\title{
Black Hole Safari: Tracking Populations and Hunting Big Game
}

\author{
Nicholas J. McConnell ${ }^{* \dagger}$ \\ Institute for Astronomy, University of Hawaii at Manoa \\ E-mail: nmcceifa.hawaii.edu
}

\begin{abstract}
Understanding the physical connection, or lack thereof, between the growth of galaxies and supermassive black holes is a key challenge in extragalactic astronomy. Dynamical studies of nearby galaxies are building a census of black hole masses across a broad range of galaxy types and uncovering statistical correlations between galaxy bulge properties and black hole masses. These local correlations provide a baseline for studying galaxies and black holes at higher redshifts. Recent measurements have probed the extremes of the supermassive black hole population and introduced surprises that challenge simple models of black hole and galaxy co-evolution. Future advances in the quality and quantity of dynamical black hole mass measurements will shed light upon the growth of massive galaxies and black holes in different cosmic environments.
\end{abstract}

Frank N. Bash Symposium 2013: New Horizons in Astronomy (BASH 2013)

October 6-8, 2013

Austin, Texas

\footnotetext{
*Speaker.

${ }^{\dagger}$ Beatrice Watson Parrent Postdoctoral Fellow
} 


\section{Supermassive Black Holes}

Broadly speaking, every massive galaxy hosts a central supermassive black hole. Lynden-Bell (1969) was among the first to note that black holes (or "Schwarzschild throats") of $10^{9}-10^{10} M_{\odot}$ could produce the tremendous energies observed in quasars and radio galaxies. More recently, observations of stars or masers at the center of the Milky Way (Schödel et al., 2002; Ghez et al., 2005), the Andromeda Galaxy (Bender et al., 2005), and NGC 4258 (Herrnstein et al., 2005) have provided unambiguous dynamical evidence for supermassive black holes. Dozens of other nearby galaxies have been found to host massive dark objects at their centers, which are assumed but not proven to be black holes. Although only a fraction of the nearest galaxies can be probed for dynamical evidence of central supermassive objects, the high detection rate suggests that they are ubiquitous in massive spheroidal systems (Gültekin et al., 2011a). Their masses, $M_{\mathrm{BH}}$, range from $\sim 10^{6} M_{\odot}$ to over $10^{10} M_{\odot}$.

The mass and density of a supermassive black hole provides a tremendous reservoir of gravitational potential energy, endowing it with the capacity to fundamentally influence the evolution of its host galaxy. Hydrodynamical simulations of galaxy formation on cosmological scales can reproduce the observed stellar mass function of galaxies only when they invoke feedback from accreting black holes to suppress star formation in massive galaxies (e.g., Croton et al., 2006; Behroozi et al., 2013). Individual systems show evidence for this feedback: massive galaxies and galaxy clusters are surrounded by a hot halo of ionized gas, which would cool and condense if not for frequent energy injection. Black holes are implicated as the energy source by radio jets extending from galaxy centers to distances well past the stellar effective radius (e.g., Perley et al., 1984), and by buoyant bubbles of gas expanding outward through the hot halos (e.g., Fabian et al., 2000).

Although supermassive black holes are a fundamental component of massive galaxies, they are arguably the least understood component. They have not been surveyed as thoroughly as stars or gas in the present-day universe, and their growth cannot be modeled as simply as the aggregation of dark matter halos. Direct observations of black holes in nearby galaxies and inferences about the black hole population fueling distant quasars are still catching up with detailed studies of galaxies throughout the universe.

\section{Black Hole Scaling Relations}

Scaling relations between black hole masses and properties of their host galaxies are a key stepping stone toward understanding supermassive black hole growth throughout the cosmos. The most reliable measurements of $M_{\mathrm{BH}}$ come from observations of stars or gas in orbit around the black hole. These dynamical measurements have typical errors $\sim 10 \%-50 \%$, but require very good spatial resolution and thus are restricted to galaxies within $\sim 100 \mathrm{Mpc}$. However, the black hole scaling relations discussed below provide a means of inferring $M_{\mathrm{BH}}$ in many more galaxies across a large range of redshifts, either by using host properties as a proxy for $M_{\mathrm{BH}}$ or by calibrating other methods for measuring $M_{\mathrm{BH}}$ in quasars and active galactic nuclei (AGN).

\subsection{Correlations in the Local Universe}

Dressler (1989) was the first to formally assert a proportional scaling relation between black 
holes and their host galaxies, based on estimates of $M_{\mathrm{BH}}$ and bulge mass ( $\left.M_{\text {bulge }}\right)$ in only five galaxies. In the following decade, the Hubble Space Telescope (HST) supplied new measurements of $M_{\mathrm{BH}}$ in over a dozen galaxies, paving the way for relations between $M_{\mathrm{BH}}$ and bulge optical luminosity ( $L$; e.g., Kormendy \& Richstone, 1995), stellar velocity dispersion ( $\sigma$; Ferrarese \& Merritt, 2000; Gebhardt et al., 2000), and an improved $M_{\mathrm{BH}}-M_{\text {bulge }}$ relation (Magorrian et al., 1998). The 2000's saw the floodgates burst open, with more measurements from HST and the emergence of adaptive optics (AO) on ground-based telescopes. The ever-growing sample of black hole masses has fueled astronomers' ambitions to discover which host galaxy property bears the tightest relation with the central black hole, resulting in a proliferation of proposed scaling relations. Multiple revisions have been made to the $M_{\mathrm{BH}}-\sigma$ relation, $M_{\mathrm{BH}}-M_{\text {bulge }}$ relation, and the $M_{\mathrm{BH}}-L$ relation in a variety of photometric bandpasses. The most recent versions of these correlations are compiled in McConnell \& Ma (2013) and Kormendy \& Ho (2013), with dynamical measurements of $M_{\mathrm{BH}}$ for more than 70 galaxies. They are illustrated in Figure 1 below. Additionally, several authors have explored relations between $M_{\mathrm{BH}}$ and total galaxy luminosity, bulge concentration or Sérsic index, the mass or corresponding circular velocity of galaxies' dark matter halos, the central stellar cores of some elliptical galaxies, and galaxies' globular cluster systems. Black hole masses appear to correlate most tightly with the bulge component of their host galaxies, with no apparent correlation between $M_{\mathrm{BH}}$ and disk mass (e.g., Kormendy \& Gebhardt, 2001). Black holes only correlate weakly with galaxy pseudobulges - compact central components with disk-like photometric and kinematic properties (Kormendy et al., 2011; Kormendy \& Ho, 2013).

A black hole scaling relation typically is defined by fitting a power law to the empirical data of $M_{\mathrm{BH}}$ versus the galaxy property of interest. This allows for straightforward predictions of $M_{\mathrm{BH}}$ in individual galaxies with (e.g.) known $\sigma$ or $L$, and for estimating the cosmic black hole mass function from well-measured quantities such as the galaxy stellar luminosity function. The empirical scaling relations at $z=0$ also provide an important baseline for assessing the relative growth of galaxies and black holes at earlier cosmic times.

\subsection{Calibrations for High-Redshift Objects}

Luminous quasars offer a window to black hole growth from as early as $770 \mathrm{Myr}$ after the Big Bang $\left(z=7.1\right.$; Mortlock et al., 2011) up to the present day. Techniques for estimating $M_{\mathrm{BH}}$ from a single quasar spectrum can be applied to large populations of objects. For instance, the Sloan Digital Sky Survey includes spectra of over 15,000 broad-line quasars (e.g., Vestergaard et al., 2008).

Unobscured quasars and AGN exhibit broad emission lines from gas orbiting the black hole at velocities $v_{\text {gas }} \sim 1000 \mathrm{~km} \mathrm{~s}^{-1}$. Although this gas is not spatially resolved, the central mass $\left(M_{\mathrm{BH}}\right)$ can be estimated by assuming Virial equilibrium:

$$
G M_{\mathrm{BH}}=f v_{\mathrm{gas}}^{2} R_{\mathrm{BLR}},
$$

where $R_{\mathrm{BLR}}$ is the orbital radius of the gas emitting broad lines, and $f$ is an unknown coefficient accounting for the geometry and orbital structure (including inflow or outflow) of the gas. In several dozen systems, $R_{\mathrm{BLR}}$ has been calculated directly by measuring time lags between continuum brightness fluctuations originating at only a few Schwarzschild radii and echoes of the same brightness fluctuations in the emission lines, a technique known as reverberation mapping (e.g., Peterson, 

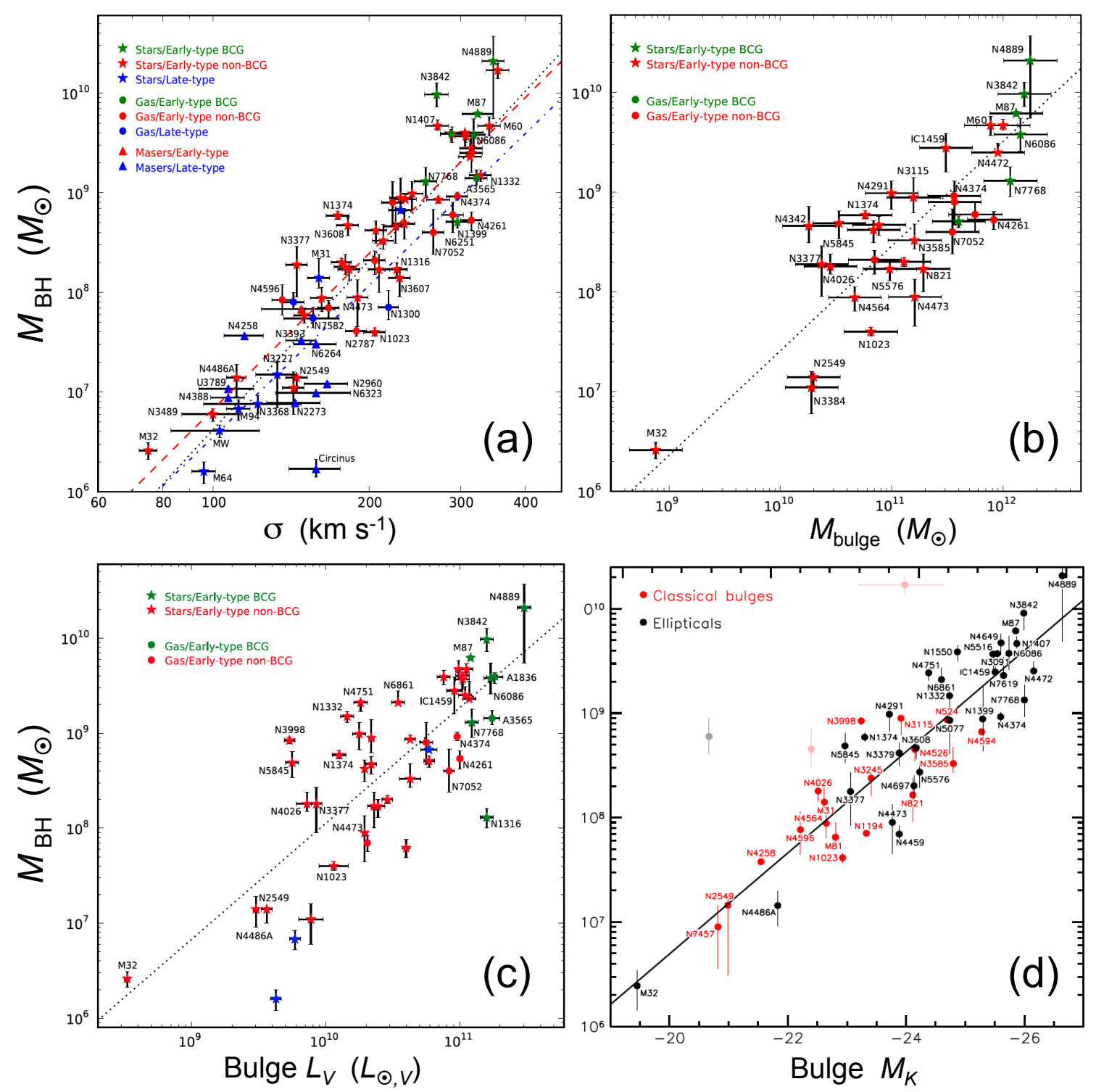

Figure 1: Recent compilations of four black hole scaling relations, based on dynamical measurements of $M_{\mathrm{BH}}$ in nearby galaxies. (a) $M_{\mathrm{BH}}-\sigma$ relation from McConnell \& Ma (2013). (b) $M_{\mathrm{BH}}-M_{\text {bulge }}$ relation from McConnell \& Ma (2013). (c) $V$-band $M_{\mathrm{BH}^{-}} L$ relation from McConnell \& Ma (2013). (d) $K$-band $M_{\mathrm{BH}^{-}}-L$ relation from Kormendy \& Ho (2013).

1993). These measurements indicate that $R_{\mathrm{BLR}}$ correlates tightly with the total quasar or AGN luminosity, which then can be used as a proxy for $R_{B L R}$ for the vast numbers of objects with spectra from only a single epoch (e.g., Kaspi et al., 2000; Greene et al., 2010b).

However, the coefficient $f$ remains an unknown parameter in estimates of quasars' $M_{\mathrm{BH}}$. This is addressed by comparing a sample of nearby AGN with known $v_{\mathrm{gas}}, R_{\mathrm{BLR}}$, and $\sigma$ to the $M_{\mathrm{BH}^{-}} \sigma$ relation derived from dynamical measurements. Although $f$ is expected to vary from galaxy to 
galaxy, the ensemble average $\langle f\rangle$ is set such that the intercept of the $M_{\mathrm{BH}^{-}} \sigma$ relation for nearby AGN matches the intercept of the dynamical $M_{\mathrm{BH}^{-}} \sigma$ relation (e.g., Onken et al., 2004; Woo et al., 2010; Park et al., 2012b). Although the Virial method for estimating $M_{\mathrm{BH}}$ is only accurate to a factor of $\sim 3$ for an individual galaxy (e.g., Park et al., 2012a), it is sufficient for assessing large samples of objects and crucial for comparing black hole masses at different redshifts. Figure 2 illustrates the upper envelope of the quasar black hole mass function. Two key features are the existence of galaxies with $M_{\mathrm{BH}}>10^{9} M_{\odot}$ at very early cosmic times, and the "downsizing" trend whereby the majority of accretion shifts from black holes of $\sim 10^{9} M_{\odot}$ at $z>1$ to lower-mass black holes at later times. Intriguingly, compilations of host galaxy properties for high-redshift quasars suggest that at fixed $M_{\mathrm{BH}}$ host galaxies were less massive at $z \geq 1$ than at present times (e.g., Peng et al., 2006a; Merloni et al., 2010). This can be interpreted as relatively early black hole growth, with bulge growth catching up later.

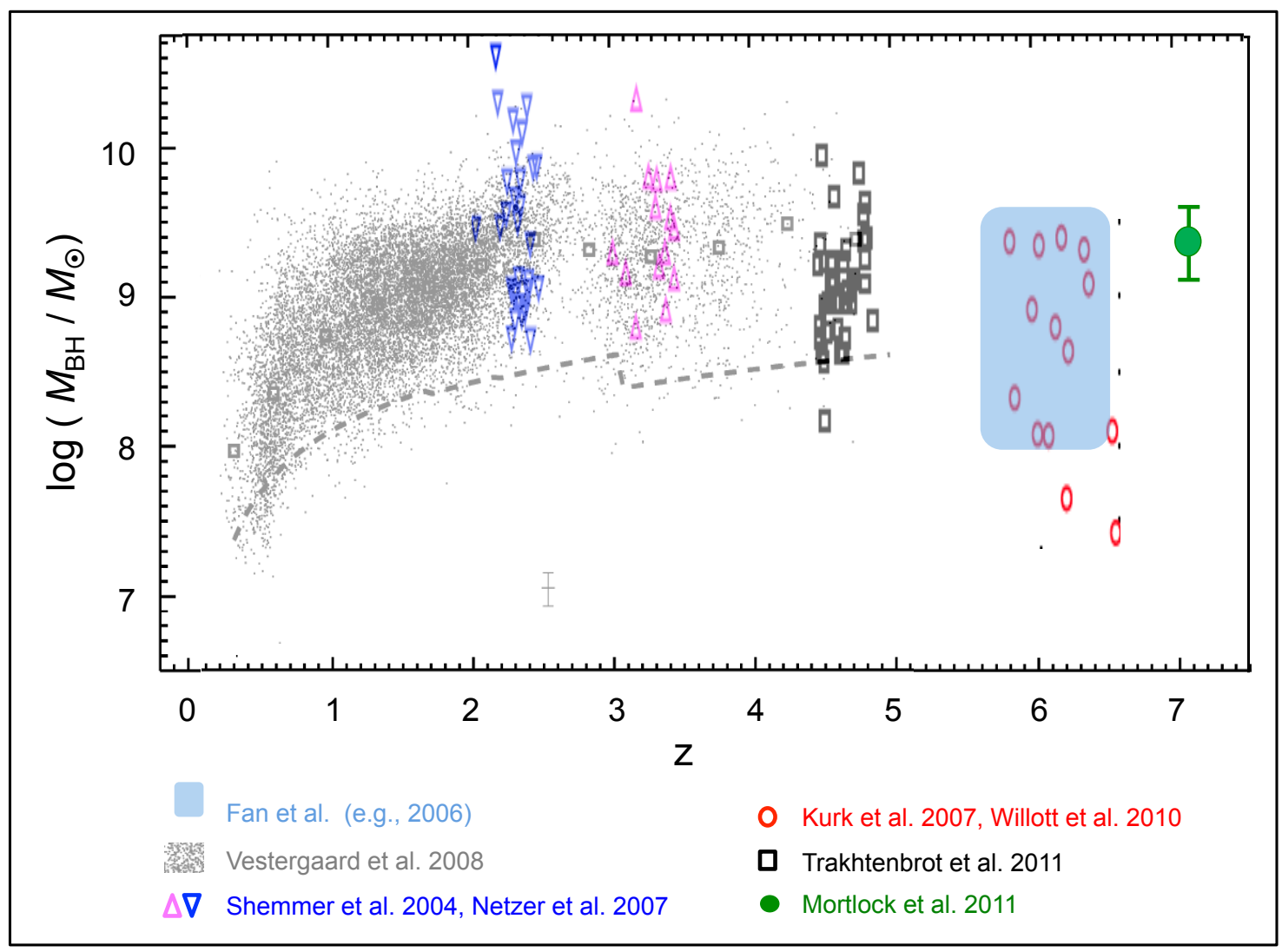

Figure 2: Compilation of $M_{\mathrm{BH}}$ for quasars, based on the Virial approximation for single-epoch spectra. The dashed line indicates the completeness limit for quasars in the Sloan Digital Sky Survey; quasars with lower $M_{\mathrm{BH}}$ likely exist but are too faint to detect. Black holes with $M_{\mathrm{BH}}>10^{9} M_{\odot}$ are observed out to $z=7$, only 770 Myr after the Big Bang. 


\subsection{Co-evolution of Galaxies and Black Holes?}

Even the largest black holes are dwarfed by their host galaxies, whose stars alone hold hundreds of times as much mass. Consequently, a black hole's gravitational dominance is confined to a small realm at the very center of the galaxy. The correlations between $M_{\mathrm{BH}}$ and large-scale galaxy properties therefore must be evolutionary in origin. Observers and theorists alike are striving to understand the physical processes that might couple galaxy and black hole growth over cosmic time.

Three broad processes can increase both $M_{\mathrm{BH}}$ and $M_{\text {bulge }}$ in a galaxy: gas-poor galaxy mergers, gas-rich galaxy mergers, and secular instabilities within a single galaxy. Gas-poor mergers are the simplest process for connecting black hole and bulge growth. To zeroth order, the final $M_{\text {bulge }}$ is simply the sum of the progenitors' $M_{\text {bulge }}$, with some adjustment for the disk-to-bulge ratio of the progenitors versus the remnant. The progenitors' supermassive black holes sink to the center of the merger remnant and coalesce, such that the final $M_{\mathrm{BH}}$ is the sum of the progenitor values.

In gas-rich mergers, gas is channeled to the center of the merger remnant where it can fuel a starburst as well as accretion onto the supermassive black hole (e.g. Sanders et al., 1988). Yet the resulting supernovae and AGN both return energy and momentum to the interstellar medium. This feedback is believed to ultimately quench star formation and black hole growth, although many details are still poorly understood (e.g., Silk \& Rees, 1998; Fabian, 1999; King, 2010a). Is stellar feedback or AGN feedback the dominant cause of quenching? Do the starburst, black hole accretion, and feedback peak at different times? Is the process that cuts off the gas supply to the black hole also responsible for driving gas out of the galaxy at larger radii? These unresolved issues, as well as variations in the gas fraction and gas properties of different progenitors, complicate whether gas-rich mergers can drive $M_{\mathrm{BH}}$ toward a common relation with galaxy properties.

Mergers are not the only way to grow $M_{\mathrm{BH}}$ and $M_{\text {bulge. In individual galaxies, secular processes }}$ such as bar instabilities can also create torques that drive gas inwards. While luminous quasars are usually associated with merging systems, lower-luminosity AGN are more common and appear to reside in a variety of hosts. Recent examinations of host galaxy morphologies for large samples of obscured and unobscured AGN have found a substantial fraction of isolated galaxies from $z=0$ to $z \sim 2$ (e.g., Cisternas et al., 2011; Schawinski et al., 2012; Treister et al., 2012). Yet we still lack precise understanding of how black hole growth is triggered in isolated galaxies, the duty cycle of accretion events, and whether subsequent feedback is quantitatively similar in individual galaxies and gas-rich mergers.

On the empirical side, the intrinsic scatter $\varepsilon_{0}$ in $M_{\mathrm{BH}}$ as a function of $\sigma$ or $M_{\text {bulge }}$ may be a key discriminant between different evolutionary scenarios. In scenarios dominated by gas-poor mergers, the $M_{\mathrm{BH}}-M_{\text {bulge }}$ relation is expected to tighten with increasing $M_{\text {bulge }}$, as successive mergers drive systems toward the cosmic average $M_{\mathrm{BH}} / M_{\text {bulge }}$ ratio (e.g., Peng, 2007). Gas-rich mergers and secular instabilities could dilute this predicted trend by introducing stochastic accretion episodes, or enhance it if the feedback mechanism strongly couples the quenching of black hole accretion to quenching star formation in the bulge. Although models with different prescriptions for black hole growth and feedback predict different trends in intrinsic scatter (e.g., Jahnke \& Macciò, 2011; Anglés-Alcázar et al., 2013), the majority of empirical studies have fit $M_{\mathrm{BH}}(\sigma)$ and $M_{\mathrm{BH}}\left(M_{\text {bulge }}\right)$ with a single value of $\varepsilon_{0}$. McConnell \& Ma (2013) attempted to track $\varepsilon_{0}$ over a few 
bins in $\sigma, L$, and $M_{\text {bulge }}$, but did not find any statistically significant trends.

Different formation scenarios for different types of galaxies may also be reflected in different black hole scaling relations. Late-type galaxies are characterized by ongoing star formation and are usually dominated by orderly rotating disks: they have relatively low $M_{\text {bulge }}$ or contain a rotating pseudobulge. Theses properties suggest the absence of catastrophic merger events in their past. Elliptical galaxies have long been envisioned as the consequence of major galaxy mergers (e.g., Toomre, 1977), but more recently they have been shown to belong to two dynamical classes: fast-rotators and slow-rotators (Emsellem et al., 2007, 2011). Connections between the kinematic properties and central surface brightness profiles of fast- and slow-rotators suggest that they differ in the gas fraction of past mergers and the number of mergers experienced (Khochfar et al., 2011; Kormendy \& Bender, 2013). If AGN feedback and star formation quenching are strongly coupled, then the final value of $M_{\mathrm{BH}}$ may be substantially higher in systems that have experienced gas-rich mergers (Zubovas \& King, 2012). However, empirical compilations of $M_{\mathrm{BH}}$ and host galaxy properties are only beginning to discern significant trends for galaxies of different types (e.g., Graham, 2012; McConnell \& Ma, 2013).

\subsection{Outliers}

Beyond the statistical scatter in the scaling relations, two galaxies have black holes at least an order of magnitude more massive than predicted from $M_{\text {bulge }}$. The black holes in NGC 1277 and NGC 4486B comprise 12\%, and 15\% of their systems' total baryonic mass, respectively (Kormendy et al., 1997; van den Bosch et al., 2012; Kormendy \& Ho, 2013). Are these galaxies examples of extreme black hole growth in the early universe, followed by starvation of the host galaxy? Interestingly, NGC 1277 and NGC 4486B are each located near the center of a galaxy cluster, yet are not the brightest cluster galaxy. This naively suggests that tidal stripping may have diminished their present-day bulge masses, but neither galaxy exhibits tidal features. It is important to learn whether these galaxies are rare individual objects, or examples of a class whose black hole masses have been poorly sampled to date.

\section{Dynamical Measurements of $M_{\mathrm{BH}}$}

The most precise measurements of $M_{\mathrm{BH}}$ rely on careful observations of stars or gas to probe orbital motions in the spatial region where the black hole's gravity dominates other sources of mass. The closest and best-studied example is the Galactic Center. Near-infrared observations spanning two decades have traced the orbits of individual stars, which are consistent with pure Keplerian motion around a central object of $4.30 \pm 0.36 \times 10^{6} M_{\odot}$ (Genzel et al., 2010). Some galaxies exhibit megamaser emission at their centers, which can be resolved with long-baseline interferometry into positions and velocities for individual masers. Keplerian motion has been detected in several maser galaxies, allowing for extremely precise measurements of $M_{\mathrm{BH}}$ (e.g., Herrnstein et al., 2005; Kuo et al., 2011).

In most galaxies, dynamical measurements of $M_{\mathrm{BH}}$ do not resolve individual point sources but rather model the line-of-sight velocity distributions (LOSVDs) of stars or gas at several locations near the galaxy center. A rule of thumb for detecting a black hole's gravitational signature is to 
resolve kinematics within the radius of influence, $r_{\mathrm{inf}} \equiv G M_{\mathrm{BH}} / \sigma^{2}$. For a galaxy on the mean $M_{\mathrm{BH}^{-}}$ $\sigma$ relation, $r_{\text {inf }} \sim 60 \mathrm{pc} \times\left(M_{\mathrm{BH}} / 10^{9} M_{\odot}\right)^{0.6}$. Spectroscopic observations with $0.1^{\prime \prime}$ resolution can detect $M_{\mathrm{BH}} \sim 10^{9} M_{\odot}$ at distances out to $\sim 100 \mathrm{Mpc}$. Stars serve as excellent dynamical tracers, as they are present in all galaxies and only feel gravitational forces. However, stellar motions are typically measured from spectroscopic absorption features, requiring high-quality data and careful template modeling. The Hubble Space Telescope and large ground-based telescopes with AO can resolve kinematics on scales of $\sim 0.1^{\prime \prime}$, but often require large integration times to attain sufficient signal-to-noise at these tiny angular scales.

Given a set of observed LOSVDs and a stellar luminosity profile, $M_{\mathrm{BH}}$ and the extended massto-light ratio are measured using orbit superposition modeling, a method introduced by Schwarzschild (1979). Orbits are computed numerically in a smooth, static gravitational potential and averaged over time to yield three-dimensional velocity profiles. A line of sight is assumed, and a weighted sum of model orbits is compared to the observed LOSVDs. Each model assumes a fixed value of $M_{\mathrm{BH}}$ and a fixed extended mass profile, often parameterized with a stellar mass-to-light ratio $\left(M_{\star} / L\right)$ plus a standard dark matter halo. The best-fit values and confidence intervals for $M_{\mathrm{BH}}$, $M_{\star} / L$, and the dark halo properties are determined by assessing the goodness-of-fit statistic $\chi^{2}$ over many models.

In some galaxies, bright emission lines trace the kinematics of an extended gas disk surrounding the black hole. Black hole mass measurements based on gas or maser emission rely on relatively simple models of a thin disk in Keplerian rotation. The enclosed mass is modeled as a black hole plus the galaxy's inner stellar mass profile, and a grid of models are run to determine the confidence intervals for $M_{\mathrm{BH}}, M_{\star} / L$, and the disk inclination. To better fit the observed kinematics, these models often include prescriptions for warps in the disk and for enhanced gas velocity dispersions (e.g., Shapiro et al., 2006; Dalla Bontà et al., 2009; Walsh et al., 2013).

\subsection{Systematic Effects}

A shortcoming of the current black hole scaling relations is that the individual measurements are heterogeneous, with different treatments for systematic errors. In fact, many of the measurements compiled in McConnell \& Ma (2013) and Kormendy \& Ho (2013) only include statistical errors. In reality, the stellar and gas dynamical methods for measuring $M_{\mathrm{BH}}$ each may be subject to a number of systematic effects.

To create accurate stellar orbit models of a galaxy, one must translate the two-dimensional light profile into a three-dimensional stellar mass profile. Thus far, almost all stellar dynamical measurements of $M_{\mathrm{BH}}$ have assumed an oblate axisymmetric stellar mass distribution. Furthermore, the effects of assuming different ellipsoid inclinations have not been rigorously tested (e.g., Gebhardt et al., 2003). Triaxial models have been developed recently, but have been tested on only a few galaxies (van den Bosch et al., 2008; van den Bosch \& de Zeeuw, 2010). In one case (NGC 3379), triaxial models yielded a two-fold increase in $M_{\mathrm{BH}}$, likely as the result of fitting box-shaped orbits that do not arise in axisymmetric systems (van den Bosch \& de Zeeuw, 2010).

Beyond geometry, most stellar dynamical models assume a constant value of $M_{\star} / L$ throughout the galaxy. In contrast, real galaxies may exhibit gradients in $M_{\star} / L$ as a result of gradients in stellar age, metallicity, or the initial mass function. McConnell et al. (2013) introduced $M_{\star} / L$ gradients into models of three giant elliptical galaxies and found that the best-fit values of $M_{\mathrm{BH}}$ changed by 
$\sim 30 \%$. Further tests with more galaxies are required to determine whether systematic errors at this level are pervasive or affect only a fraction of galaxies with stellar kinematic data.

Models of nuclear gas disks also require assumptions about the disk geometry. Whereas simple models assume a flat, continuous disk, the observed kinematics may be altered by warps or non-circular motions (e.g., Shapiro et al., 2006), or by an inner hole truncating the disk emission (Macchetto et al., 1997). Perhaps more insidious are possible effects from non-gravitational forces such as radiation pressure and turbulence. Many nuclear disks exhibit emission line widths that are too large to match the observed rotation profiles (e.g., Verdoes Kleijn et al., 2002; Shapiro et al., 2006; Dalla Bontà et al., 2009; Walsh et al., 2013). Disk models typically are modified to incorporate excess line widths, but uncertainty about their physical origin warrants concern that other elements of the gas physics have been oversimplified.

Only a handful of galaxies have had $M_{\mathrm{BH}}$ measured using multiple dynamical tracers. The independent measurements are broadly consistent for NGC 5128 (Neumayer et al., 2007; Cappellari et al., 2009) and NGC 4258 (Siopis et al., 2009), whereas there are significant discrepancies for IC 1459 (Cappellari et al., 2002) and NGC 3998 (Walsh et al., 2012). The gas dynamical measurement of $M_{\mathrm{BH}}$ in NGC 3379 is consistent with $M_{\mathrm{BH}}$ from axisymmetric stellar models (Shapiro et al., 2006) but discrepant with the more recent triaxial result (van den Bosch \& de Zeeuw, 2010). Of particular interest is the giant elliptical M87, which was first proposed to show dynamical evidence for a supermassive black hole by Sargent et al. (1978). Today, M87 has stellar kinematic measurements on scales of 6 pc (from Gemini NIFS; Gebhardt et al., 2011) and a nuclear gas disk resolved at $8 \mathrm{pc}$ with HST (Walsh et al., 2013). Both stellar and gas data boast resolution $<0.1 r_{\text {inf }}$. Yet stellar dynamical measurements of $M_{\mathrm{BH}}$ are consistently $\sim 2 \times$ higher than gas dynamical measurements, as illustrated in Figure 3. This discrepancy likely results from uncorrected systematic errors in one or both of the stellar and gas dynamical models of M87.

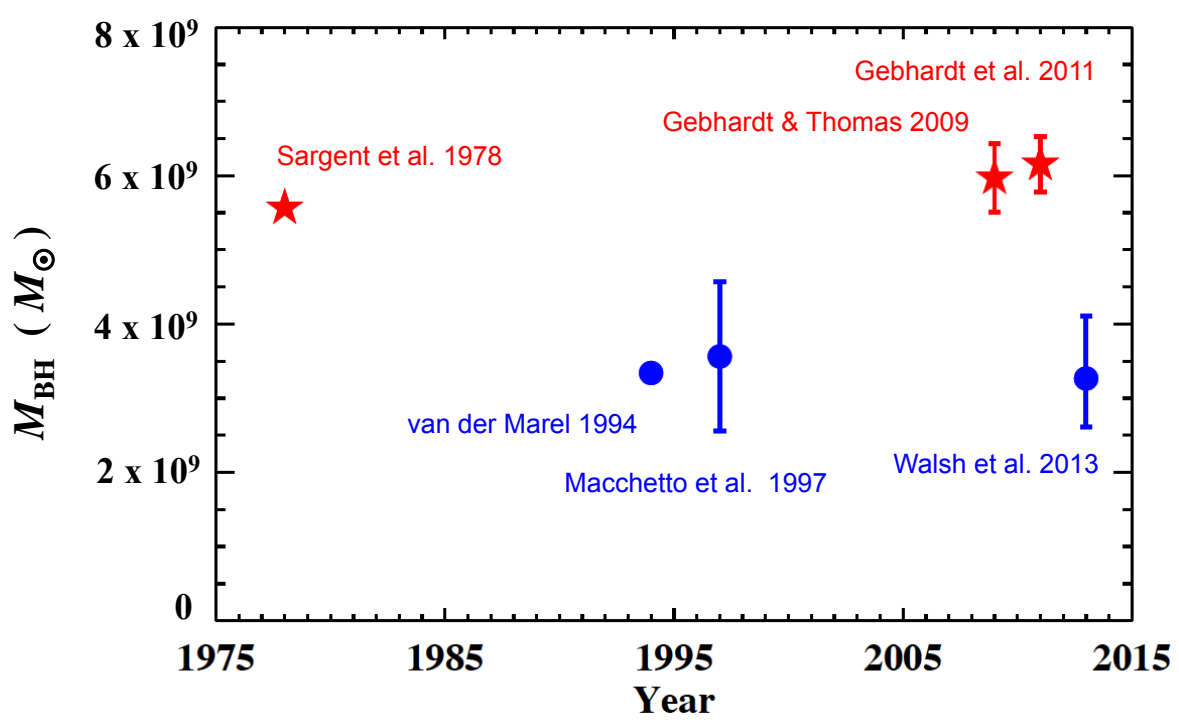

Figure 3: Dynamical measurements of $M_{\mathrm{BH}}$ in M87. Blue circles represent measurements using gas dynamics, and red stars represent measurements using stellar dynamics. 


\section{The Black Hole Safari}

Our understanding of supermassive black holes can greatly benefit from a larger sample of dynamically measured black hole masses, spanning a larger range of $M_{\mathrm{BH}}$. For instance, competing models for the cosmic abundance and initial mass of supermassive black hole seeds predict notably different trends in $M_{\mathrm{BH}}$ for present-day galaxies with $\sigma<100 \mathrm{~km} \mathrm{~s}^{-1}$ (e.g., Volonteri et al., 2008; Volonteri \& Natarajan, 2009). Unfortunately, these galaxies are predicted to host black holes with $M_{\mathrm{BH}}<10^{7} M_{\odot}$, whose gravitational influence on surrounding stars is presently undetectable at distances beyond a few Mpc. At the opposite end, accurate estimates of the black hole mass function out to $M_{\mathrm{BH}} \sim 10^{9}-10^{10} M_{\odot}$ are needed to determine the space densities and duty cycles of the most energetic quasars and radio galaxies. Yet the large distances and faint centers of extremely luminous galaxies present observational challenges for directly measuring $M_{\mathrm{BH}}$. Additional goals for future investigations are to constrain the number of extreme objects like NGC 1277 and NGC 4486B, and to reduce systematic errors in individual measurements of $M_{\mathrm{BH}}$, thereby improving the statistical analysis of correlations between black holes and host galaxy properties.

The Black Hole Safari is an ambitious campaign to measure $\sim 40$ new black hole masses in a diverse sample of massive early-type galaxies. This will double the number of early-type galaxies with dynamical measurements of $M_{\mathrm{BH}}$. The survey is designed to enable clear comparisons between black hole growth in cluster versus group environments. To this end, our sample includes over a dozen brightest cluster galaxies and over a dozen brightest group galaxies. Thus far we have observed 25 galaxies, shown in Figure 4.

Galaxy clusters represent extreme peaks in cosmic mass density, and their progenitors in the early universe were plausible sites for the birth and rapid growth of supermassive black hole seeds. However, gas depletion of galaxies falling into massive clusters could inhibit black hole accretion at later times, and the environmental dependence of black hole growth is still unclear. For instance, McGee (2013) offers preliminary evidence that central group galaxies obey steeper $M_{\mathrm{BH}^{-}} \sigma$ and $M_{\mathrm{BH}}-M_{\text {bulge }}$ relations than satellite galaxies, but a larger sample is necessary to detect a decisive trend. The Black Hole Safari will also place better constraints upon the cosmic scatter in $M_{\mathrm{BH}}$ for the most massive elliptical galaxies, which indicates the relative importance of stochastic accretion versus hierarchical merging (e.g., Hirschmann et al., 2010; Jahnke \& Macciò, 2011). The survey will also improve constraints upon the number density of objects with extreme $M_{\mathrm{BH}} / M_{\text {bulge }}$ ratios.

Black hole masses will be measured using stellar dynamics, based on integral-field spectroscopic (IFS) data from ground-based telescopes. At least half of the galaxies in the Black Hole Safari sample have predicted $r_{\text {inf }}>0.4^{\prime \prime}$ based on their values of $\sigma$ and $L$. This will allow $M_{\mathrm{BH}}$ to be measured with seeing-limited data from GMOS on Gemini North or South: queue-mode observations on nights with high image quality allow us to take advantage of premier observing sites at Mauna Kea and Cerro Pachón. Other targets require AO observations to attain spatial resolution of $\approx 0.1^{\prime \prime}$. We will observe these objects with NIFS on Gemini North and OSIRIS on Keck 1.

While high-resolution IFS observations will provide crucial data for measuring $M_{\mathrm{BH}}$, widefield observations are necessary to alleviate degeneracies between stellar mass and dark matter in the stellar dynamical models. Fifteen targets from the Black Hole Safari have existing data from the Mitchell Spectrograph (formerly VIRUS-P), an IFS on the 2.7-m telescope at McDonald Observatory with a field-of-view of $107^{\prime \prime}$. Finally, high-resolution photometry is necessary to constrain 


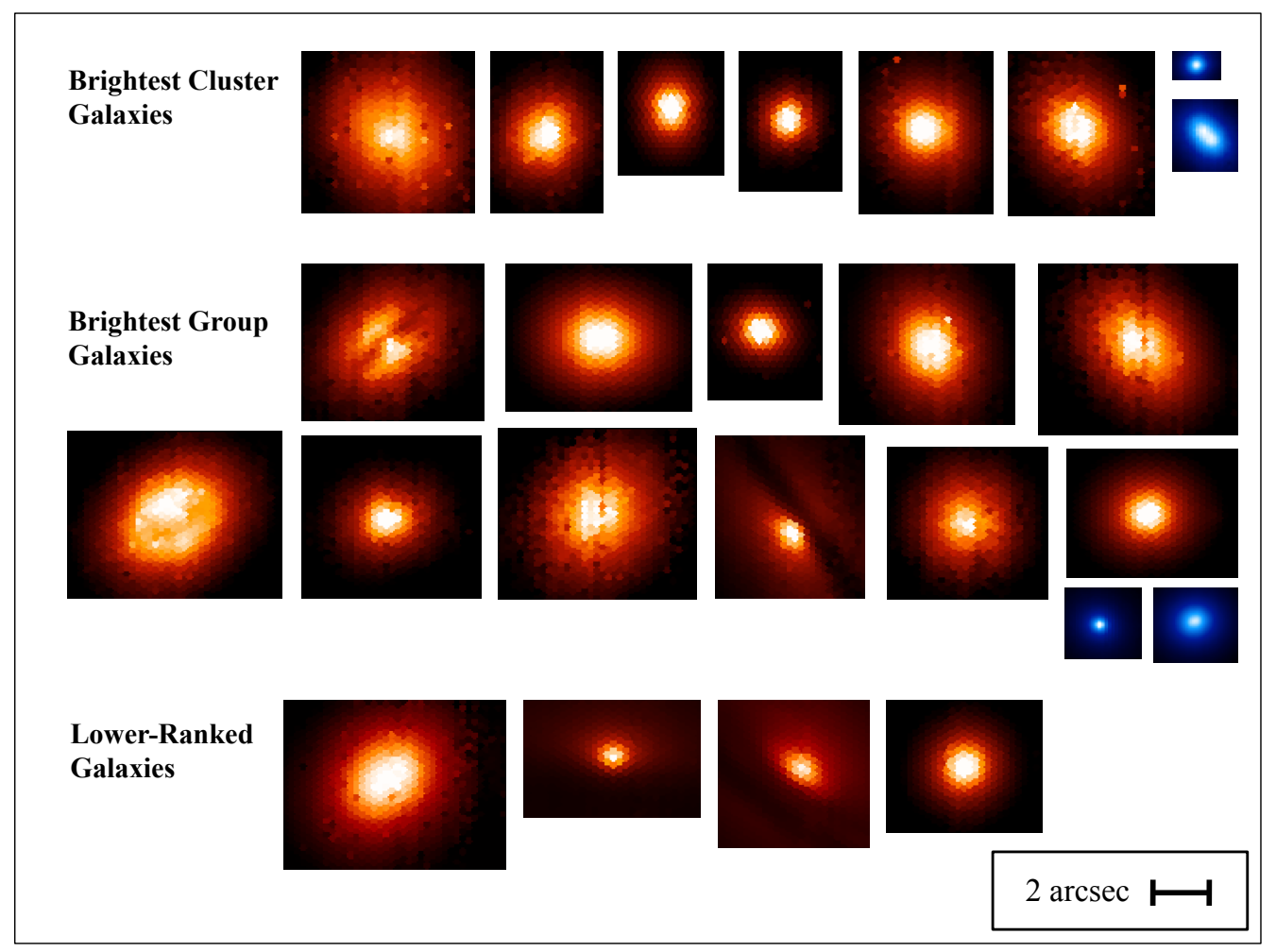

Figure 4: IFS maps of total flux in the centers of eight BGCs, 13 brightest group galaxies, and four other galaxies in the Black Hole Safari survey. The majority of the data are from the GMOS instruments on Gemini North and Gemini South, while the four smallest boxes (shaded in blue) are from NIFS on Gemini North and OSIRIS on Keck. Five BCGs with published measurements of $M_{\mathrm{BH}}$ (McConnell et al., 2011a, 2012) are not shown.

the inner stellar mass profile of each galaxy. Most of the Black Hole Safari galaxies have archival HST data (e.g., Lauer et al., 2005).

As all of the Black Hole Safari observations use integral-field spectroscopy, this survey is ideally suited for assessing correlations between black hole masses and detailed structural properties of galaxies. Examples include comparing $M_{\mathrm{BH}}$ in fast- and slow-rotating galaxies (e.g., Emsellem et al., 2011) and analyzing the quantitative relationship between $M_{\mathrm{BH}}$ and the mass or size of stellar cusps and cores at the centers of early-type galaxies (Kormendy \& Bender, 2009; Rusli et al., 2013b).

\section{Conclusion}

Investigations of galaxy dynamics in the next few years have potential to solidify or befuddle our basic notions of black hole and galaxy co-evolution, even before new telescopes bring further advances in spatial resolution. Steady increases in the sample size and diversity of galaxies hosting accurately measured $M_{\mathrm{BH}}$ are already providing ample food for thought. The three most massive 
black holes known were discovered just in the past three years (McConnell et al., 2011b; van den Bosch et al., 2012), and surveys such as the Black Hole Safari are expanding the search to dozens of similar targets. In reporting the anomalously large black hole in NGC 1277, van den Bosch et al. (2012) noted several other galaxies with preliminary hints of extreme $M_{\mathrm{BH}} / M_{\text {bulge }}$ ratios. Will these objects or others upend the black hole scaling relations as we know them? On the theoretical side, the processes that drive gas down toward black holes and deposit energy from accretion back into the ISM are being modeled with increasing sophistication. The notion of global scaling relations for all galaxies and black holes is on precarious footing, but empirical and theoretical studies may soon be able to distinguish different evolutionary scenarios for different galaxy types.

While the number of black hole mass measurements in nearby galaxies is certain to increase, the quality of dynamical modeling methods deserves close scrutiny as well. Stellar and gas dynamical methods both have lingering systematic effects, whose leverage on individual measurements and the overall scaling relations is not yet clear. As new observations amass, one hopes they will be disseminated transparently enough to enable further testing and revision as methods improve.

\section{References}

Anglés-Alcázar, D., Özel, F., \& Davé, R. 2013, ApJ, 770, 5

Behroozi, P. S., Wechsler, R. H., \& Conroy, C. 2013, ApJ, 770, 57

Bender, R., Kormendy, J., Bower, G., et al. 2005, ApJ, 631, 280

Cappellari, M., Neumayer, N., Reunanen, J., et al. 2009, MNRAS, 394, 660

Cappellari, M., Verolme, E. K., van der Marel, R. P., et al. 2002, ApJ, 578, 787

Cisternas, M., Jahnke, K., Inskip, K. J., et al. 2011, ApJ, 726, 57

Croton, D. J., Springel, V., White, S. D. M., et al. 2006, MNRAS, 365, 11

Dalla Bontà, E., Ferrarese, L., Corsini, E. M., et al. 2009, ApJ, 690, 537

Dressler, A. 1989, in IAU Symposium, Vol. 134, Active Galactic Nuclei, ed. D. E. Osterbrock \& J. S. Miller, 217

Emsellem, E., Cappellari, M., Krajnović, D., et al. 2007, MNRAS, 379, 401

-. 2011, MNRAS, 414,888

Fabian, A. C. 1999, MNRAS, 308, L39

Fabian, A. C., Sanders, J. S., Ettori, S., et al. 2000, MNRAS, 318, L65

Fan, X., Strauss, M. A., Becker, R. H., et al. 2006, AJ, 132, 117

Ferrarese, L., \& Merritt, D. 2000, ApJ, 539, L9

Gebhardt, K., Adams, J., Richstone, D., et al. 2011, ApJ, 729, 119 
Gebhardt, K., \& Thomas, J. 2009, ApJ, 700, 1690

Gebhardt, K., Bender, R., Bower, G., et al. 2000, ApJ, 539, L13

Gebhardt, K., Richstone, D., Tremaine, S., et al. 2003, ApJ, 583, 92

Genzel, R., Eisenhauer, F., \& Gillessen, S. 2010, Reviews of Modern Physics, 82, 3121

Ghez, A. M., Salim, S., Hornstein, S. D., et al. 2005, ApJ, 620, 744

Graham, A. W. 2012, ApJ, 746, 113

Greene, J. E., Hood, C. E., Barth, A. J., et al. 2010b, ApJ, 723, 409

Gültekin, K., Tremaine, S., Loeb, A., \& Richstone, D. O. 2011a, ApJ, 738, 17

Herrnstein, J. R., Moran, J. M., Greenhill, L. J., \& Trotter, A. S. 2005, ApJ, 629, 719

Hirschmann, M., Khochfar, S., Burkert, A., et al. 2010, MNRAS, 407, 1016

Jahnke, K., \& Macciò, A. V. 2011, ApJ, 734, 92

Kaspi, S., Smith, P. S., Netzer, H., et al. 2000, ApJ, 533, 631

Khochfar, S., Emsellem, E., Serra, P., et al. 2011, MNRAS, 417, 845

King, A. R. 2010a, MNRAS, 402, 1516

Kormendy, J., \& Bender, R. 2009, ApJ, 691, L142

-. 2013, ApJ, 769, L5

Kormendy, J., Bender, R., \& Cornell, M. E. 2011, Nature, 469, 374

Kormendy, J., \& Gebhardt, K. 2001, in American Institute of Physics Conference Series, Vol. 586, 20th Texas Symposium on relativistic astrophysics, ed. J. C. Wheeler \& H. Martel, 363-381

Kormendy, J., \& Ho, L. C. 2013, ARAA, 51, 511

Kormendy, J., \& Richstone, D. 1995, ARAA, 33, 581

Kormendy, J., Bender, R., Magorrian, J., et al. 1997, ApJ, 482, L139

Kuo, C. Y., Braatz, J. A., Condon, J. J., et al. 2011, ApJ, 727, 20

Kurk, J. D., Walter, F., Fan, X., et al. 2007, ApJ, 669, 32

Lauer, T. R., Faber, S. M., Gebhardt, K., et al. 2005, AJ, 129, 2138

Lynden-Bell, D. 1969, Nature, 223, 690

Macchetto, F., Marconi, A., Axon, D. J., et al. 1997, ApJ, 489, 579 
Magorrian, J., Tremaine, S., Richstone, D., et al. 1998, AJ, 115, 2285

McConnell, N. J., Chen, S.-F. S., Ma, C.-P., et al. 2013, ApJ, 768, L21

McConnell, N. J., \& Ma, C.-P. 2013, ApJ, 764, 184

McConnell, N. J., Ma, C.-P., Gebhardt, K., et al. 2011b, Nature, 480, 215

McConnell, N. J., Ma, C.-P., Graham, J. R., et al. 2011a, ApJ, 728, 100

McConnell, N. J., Ma, C.-P., Murphy, J. D., et al. 2012, ApJ, 756, 179

McGee, S. L. 2013, MNRAS, 436, 2708

Merloni, A., Bongiorno, A., Bolzonella, M., et al. 2010, ApJ, 708, 137

Mortlock, D. J., Warren, S. J., Venemans, B. P., et al. 2011, Nature, 474, 616

Netzer, H., Lira, P., Trakhtenbrot, B., Shemmer, O., \& Cury, I. 2007, ApJ, 671, 1256

Neumayer, N., Cappellari, M., Reunanen, J., et al. 2007, ApJ, 671, 1329

Onken, C. A., Ferrarese, L., Merritt, D., et al. 2004, ApJ, 615, 645

Park, D., Kelly, B. C., Woo, J.-H., \& Treu, T. 2012b, ApJS, 203, 6

Park, D., Woo, J.-H., Treu, T., et al. 2012a, ApJ, 747, 30

Peng, C. Y. 2007, ApJ, 671, 1098

Peng, C. Y., Impey, C. D., Ho, L. C., Barton, E. J., \& Rix, H.-W. 2006a, ApJ, 640, 114

Perley, R. A., Dreher, J. W., \& Cowan, J. J. 1984, ApJ, 285, L35

Peterson, B. M. 1993, PASP, 105, 247

Rusli, S. P., Erwin, P., Saglia, R. P., et al. 2013b, AJ, 146, 160

Sanders, D. B., Soifer, B. T., Elias, J. H., et al. 1988, ApJ, 325, 74

Sargent, W. L. W., Young, P. J., Lynds, C. R., et al. 1978, ApJ, 221, 731

Schawinski, K., Simmons, B. D., Urry, C. M., Treister, E., \& Glikman, E. 2012, MNRAS, 425, L61

Schödel, R., Ott, T., Genzel, R., et al. 2002, Nature, 419, 694

Schwarzschild, M. 1979, ApJ, 232, 236

Shapiro, K. L., Cappellari, M., de Zeeuw, T., et al. 2006, MNRAS, 370, 559

Shemmer, O., Netzer, H., Maiolino, R., et al. 2004, ApJ, 614, 547 
Silk, J., \& Rees, M. J. 1998, A\&A, 331, L1

Siopis, C., Gebhardt, K., Lauer, T. R., et al. 2009, ApJ, 693, 946

Toomre, A. 1977, in Evolution of Galaxies and Stellar Populations, ed. B. M. Tinsley \& R. B. G. Larson, D. Campbell, 401

Trakhtenbrot, B., Netzer, H., Lira, P., \& Shemmer, O. 2011, ApJ, 730, 7

Treister, E., Schawinski, K., Urry, C. M., \& Simmons, B. D. 2012, ApJ, 758, L39

van den Bosch, R. C. E., \& de Zeeuw, P. T. 2010, MNRAS, 401, 1770

van den Bosch, R. C. E., Gebhardt, K., Gültekin, K., et al. 2012, Nature, 491, 729

van den Bosch, R. C. E., van de Ven, G., Verolme, E. K., Cappellari, M., \& de Zeeuw, P. T. 2008, MNRAS, 385, 647

van der Marel, R. P. 1994, MNRAS, 270, 271

Verdoes Kleijn, G. A., van der Marel, R. P., de Zeeuw, P. T., Noel-Storr, J., \& Baum, S. A. 2002, $\mathrm{AJ}, 124,2524$

Vestergaard, M., Fan, X., Tremonti, C. A., Osmer, P. S., \& Richards, G. T. 2008, ApJ, 674, L1

Volonteri, M., Lodato, G., \& Natarajan, P. 2008, MNRAS, 383, 1079

Volonteri, M., \& Natarajan, P. 2009, MNRAS, 400, 1911

Walsh, J. L., Barth, A. J., Ho, L. C., \& Sarzi, M. 2013, ApJ, 770, 86

Walsh, J. L., van den Bosch, R. C. E., Barth, A. J., \& Sarzi, M. 2012, ApJ, 753, 79

Willott, C. J., Albert, L., Arzoumanian, D., et al. 2010, AJ, 140, 546

Woo, J.-H., Treu, T., Barth, A. J., et al. 2010, ApJ, 716, 269

Zubovas, K., \& King, A. R. 2012, MNRAS, 426, 2751 